\title{
A Full-Field Tomographic Imaging Camera Based on a Linearly Swept Frequency DFB at 1064 nm
}

\author{
Mark Harfouche ${ }^{1, *}$, Naresh Satyan ${ }^{2}$, George Rakuljic ${ }^{2}$, and Amnon Yariv ${ }^{1,3}$ \\ ${ }^{1}$ Department of Electrical Engineering, California Institute of Technology, 1200 E. California Blvd., Pasadena, CA \\ 91125, USA \\ ${ }^{2}$ Telaris Inc., 2118 Wilshire Blvd. \#238, Santa Monica, CA 90403, USA \\ ${ }^{3}$ Department of Applied Physics and Materials Science, California Institute of Technology, 1200 E. California Blvd., \\ Pasadena, CA 91125, USA \\ ${ }^{*}$ Corresponding author: mark.harfouche@ caltech.edu
}

\begin{abstract}
High resolution, full-field tomograms are acquired in four exposures of a CCD camera using a swept laser. The imaged depth is selected by modulating the swept laser output power enabling volumetric imaging with no moving parts.
\end{abstract}

(C) 2014 Optical Society of America

OCIS codes: (110.4500) Optical coherence tomography, (110.6955) Tomographic imaging.

Optical coherence tomography has become an important high resolution 3D imaging modality for many biomedical applications [1]. However, many embodiments require a mechanical scanning element to change the lateral imaging location [2] or the imaging depth [3]. Here, we propose and demonstrate the acquisition of tomograms-all lateral locations $(x, y)$ at a constant depth $(z)$-using principles inspired from swept source optical coherence tomography (SSOCT). Changing the imaged depth $(z)$ is achieved by varying the electrical driving frequency of an intensity modulator while the lateral information is captured in parallel with a CCD camera. Combining the depth information from multiple tomographic slices in software allows full 3D reconstruction of the scene. The proposed method overcomes the requirement that the system be interferometrically stable for the entire duration of the 3D scan since only 4 consecutive frames are needed to measure a particular tomogram.

SSOCT measures the beat frequency of interference between the reflected light from a sample and a reference wave on a photodetector when both are illuminated by a chirped laser. The measured current is given by

$$
i(x, y, t) \propto P_{0}+2 \sqrt{P_{0} P_{s}\left(x, y, \tau_{s}\right)} \cos \left(\xi \tau_{s}+\phi_{s}\right),
$$

where $P_{0}$ is the received power from the reference wave, $P_{\mathrm{s}}\left(x, y, \tau_{s}\right) \ll P_{0}$ is the received power from the scene at the imaged lateral location. The frequency of the electrical signal $\left(v_{\mathrm{s}}=\xi \tau_{\mathrm{s}}\right)$ is proportional to the chirp rate $(\xi)$ and the optical delay $\left(\tau_{\mathrm{s}}\right)$ between the sample and the reference. The phase of the beat signal $\left(\phi_{\mathrm{s}}\right)$ contains information about the sub-resolution position (wavelength scale) of the sample and is typically ignored. The measurement of this beat signal across a dense focal plane array is impractical due to the high sampling rates required to measure it, typically a few MHz per pixel. Here, a single modulator is used to electronically shift the high frequency content to DC where parallel measurements are readily achieved with a camera.

When the intensity of the swept laser is varied sinusoidally, the modulation beats with the the high frequency information creating a sideband at DC. Since the information of a particular depth is now encoded in the average received power, it can be measured with an array of slow photodetectors such as a CCD camera. The frequency of the electrical modulation therefore selects the depth at which the received power from the scene is measured on the camera. For weak reflectors each pixel $(x, y)$ on the camera measures

$$
N\left(x, y, v_{\mathrm{mod}}, \phi_{\mathrm{mod}}\right) \propto \int_{0}^{T} d t\left[1+\cos \left(v_{\mathrm{mod}} t+\phi_{\mathrm{mod}}\right)\right] \cdot i(x, y, t) \approx P_{0}+\cos \left(\phi_{\mathrm{mod}}-\phi_{\mathrm{s}}\right) \sqrt{P_{\mathrm{ref}} P_{\mathrm{s}}\left(x, y, z=\frac{v_{\mathrm{mod}} c}{2 \xi}\right)},
$$

where $v_{\text {mod }}$ and $\phi_{\text {mod }}$ are the frequency and phase of the intensity modulation, $T$ is the chirp duration, and $c$ is the speed of light. Reflections from all other depths $\left(z \neq \frac{v_{\bmod } c}{2 \xi}\right)$ average to zero and are not measured by the camera. The signal, $P_{\mathrm{S}}(x, y, z)$, can be isolated from the average received power, $P_{0}$, in four measurements by changing the electrical phase of the modulation through

$$
P_{0} P_{\mathrm{s}}\left(x, y, z=\frac{v_{\mathrm{mod}} c}{2 \xi}\right) \propto\left[N\left(x, y, v_{\mathrm{mod}}, 0\right)-N\left(x, y, v_{\mathrm{mod}}, \pi\right)\right]^{2}+\left[N\left(x, y, v_{\mathrm{mod}}, \pi / 2\right)-N\left(x, y, v_{\mathrm{mod}}, 3 \pi / 2\right)\right]^{2},
$$


and will be peaked when $v_{\text {mod }}=\xi \tau_{s} . P_{0}$ can be estimated from the average of the four (4) aforementioned frames. Volumetric imaging is achieved by varying the frequency of the modulation over the range of interest. The linearity and starting wavelength of the optical sweep is ensured by locking the chirp rate to an electronic reference using the feedback scheme described in [4]. This enables measurements from consecutive exposures to be added coherently.

(a)

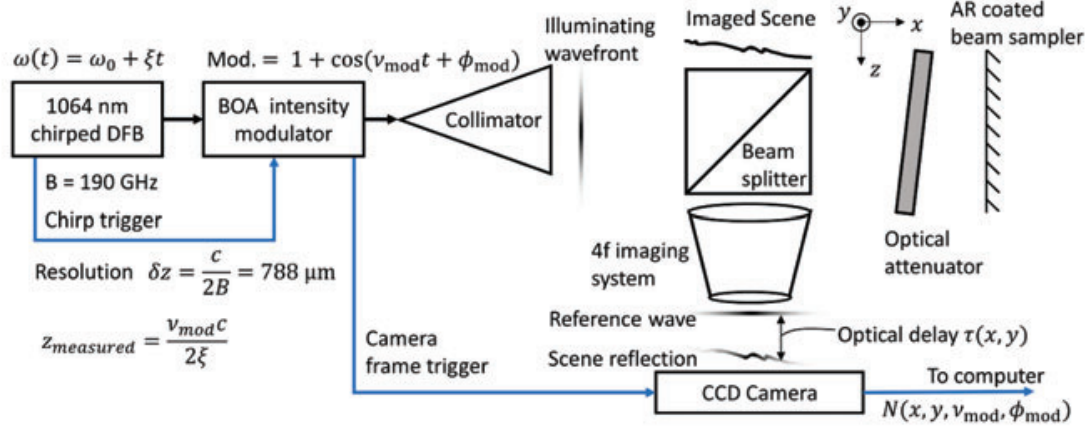

(b) Illuminated region with

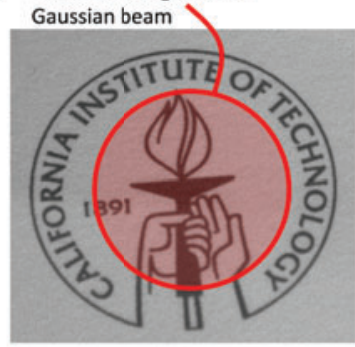

Fig. 1. (a) The imaging system setup depicting the $1064 \mathrm{~nm}$ chirped distributed feedback laser (DFB), the fiber coupled booster optical amplifier (BOA) used as an intensity modulator, and the free space Michelson interferometer. The reference wavefront is a reflection from a wedge with an anti-reflection (AR) coating. $\omega(t)$ denotes the optical frequency of laser. (b) The imaged scene illuminated with white light showing the region that will be illuminated with the described scheme.

To demonstrate the performance of this imaging modality, the imaged sample is placed in one arm of a free space Michelson interferometer and a partially reflecting mirror in the other, as shown in Fig. 1(a). The sample, Fig. 1(b), consists of the Caltech emblem on a tilted business card tilted, see Fig. 2(a). The imaging setup includes a 4-f system, with a lateral resolution of approximately $100 \mu \mathrm{m}$, to simultaneously image the reference wave as well as the scene on the camera. Each frame averages the signal received from $251.8 \mathrm{~ms}$ chirps from a $1064 \mathrm{~nm}$ DFB modulated with the same waveform illuminate over $100 \mathrm{~ms}$. The dead time between chirps $(2.2 \mathrm{~ms})$ is necessary to ensure identical laser sweeps. Note, only a single sweep per exposure is required given increased average output power from the modulator, currently $\sim 25 \mathrm{~mW}$. A particular tomogram acquired in 4 consecutive exposures, Fig. 2(b), shows the depth selectivity of the system. Fig. 2(c) shows the measured signal at 3 different locations as the interrogated depth is varied from 10 to $50 \mathrm{~mm}$. Each location on the paper appears at a single resolution limited depth $(\delta z=788 \mu \mathrm{m})$. The axial resolution of the system is determined by the bandwidth of the chirp, here $B=190 \mathrm{GHz}$, identical to that of conventional SSOCT.
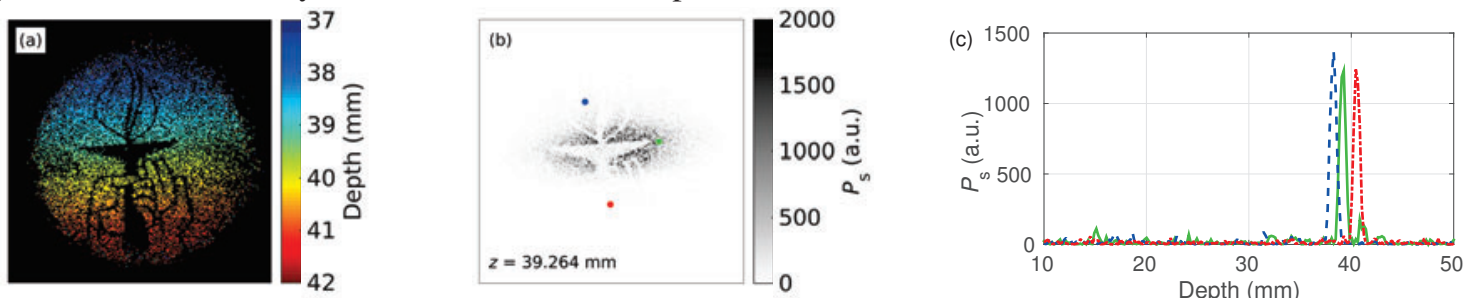

Fig. 2. (a) Surface profile of the card measured by plotting the depth location of the signal peak. Black denotes regions where the signal is less than $P_{0} / 10$ to outline the logo. (b) Tomogram $(250 \times 250$ pixels $)$ of the business card showing only the middle section of the logo. (c) Received signal power as a function of depth at the three lateral locations highlighted in (b).

The authors would like to acknowledge the support of DARPA through the KECoM program and useful discussions with Arseny Vasilyev.

\section{References}

1. S. A. Boppart, M. E. Brezinski, B. E. Bouma, G. J. Tearney, and J. G. Fujimoto, "Investigation of developing embryonic morphology using optical coherence tomography," Developmental Biology 177, 54 - 63 (1996).

2. R. Huber, M. Wojtkowski, and J. G. Fujimoto, "Fourier Domain Mode Locking (FDML): A new laser operating regime and applications for optical coherence tomography." Optics express 14, 3225-37 (2006).

3. H. M. Subhash, "Full-Field and Single-Shot Full-Field Optical Coherence Tomography: A Novel Technique for Biomedical Imaging Applications," Advances in Optical Technologies 2012, 1-26 (2012).

4. N. Satyan, A. Vasilyev, G. Rakuljic, V. Leyva, and A. Yariv, "Precise control of broadband frequency chirps using optoelectronic feedback," Opt. Express 17, 15,991-15,999 (2009). 